\title{
DÉBIT D'ÉCHANTILLONS D'ÉPAISSEUR RIGOUREUSEMENT UNIFORME A L'AIDE DE SCIES-FRAISES JUMELÉES
}

\author{
F. THIERCELIN et J. R. PERRIN \\ Station de Recherches sur la Qualité des Bois \\ Centre national de Recherches forestières \\ 54 - Champenoux
}

L'obtention d'échantillons d'épaisseur uniforme à partir d'éprouvettes de formes variées peut s'avérer nécessaire dans un certain nombre d'applications technologiques importantes :

- étude de rétractibilité sur échantillons parallélépipédiques de taille inférieure aux éprouvettes normalisées,

- analyse densitométrique dans les cas où l'utilisation des carottes de sondage brutes n'est pas possible,

- étude de longueur de fibres sur carottes obliques avec enlèvement des dosses où le pourcentage de fibres coupées est trop élevé.

Ceci nous a conduits à développer depuis 1966 différents types de sciages de précision. La méthode décrite ci-dessous est maintenant parfaitement fonctionnelle et, grâce à l'automatisation des opérations, donne des résultats satisfaisants, quel que soit l'opérateur.

\section{MATÉRIEL}

Sur un tour équipé d'un dispositif de fraisage vertical, l'échantillon est fixé sur le chariot porteoutil à tourelle orientable dont le déplacement est assuré par la vis-mère du tour.

L'outil consiste en deux scies-fraises de $63 \mathrm{~mm}$ de diamètre séparées par une rondelle dont l'épaisseur est égale à celle du sciage désiré; pour le débit des carottes de sondage, on utilise des scies de $0,5 \mathrm{~mm}$ d'épaisseur au pas de $2 \mathrm{~mm}$; pour le découpage d'échantillons parallélépipédiques plus épais, on emploie de préférence des scies-fraises de $1 \mathrm{~mm}$ d'épaisseur au pas de 1,55 $\mathrm{mm}$.

Le dispositif de sciage est fixé dans la tête de fraisage, de façon que le débit soit fait parrallèlement au déplacement du chariot. La vitesse de rotation de l'outil (en général 350 tours/minute) et la vitesse d'avancement du chariot (de $40 \mathrm{~mm} /$ minute pour les bois tendres à $17 \mathrm{~mm} /$ minute pour les bois durs) sont choisies de façon à obtenir la plus grande vitesse de sciage possible, sans effet d'échauffement préjudiciable au bon fonctionnement des scies-fraises.

De plus, l'utilisation d'un système d'aspiration réduit ce risque d'échauffement en éliminant la sciure au fur et à mesure de sa formation.

\section{MODE OPÉRATOIRE}

\section{I. - Cas des carottes de sondage de $5 \mathrm{~mm}$ de diamètre : (figures 1 et 2)}

Bien que le matériel de base de l'analyse densitométrique soit constitué par des carottes de $5 \mathrm{~mm}$ brutes de prélèvement, le débit d'échantillons d'épaisseur constante peut s'imposer, aussi bien en radiographie axiale qu'en radiographie tangentielle : 


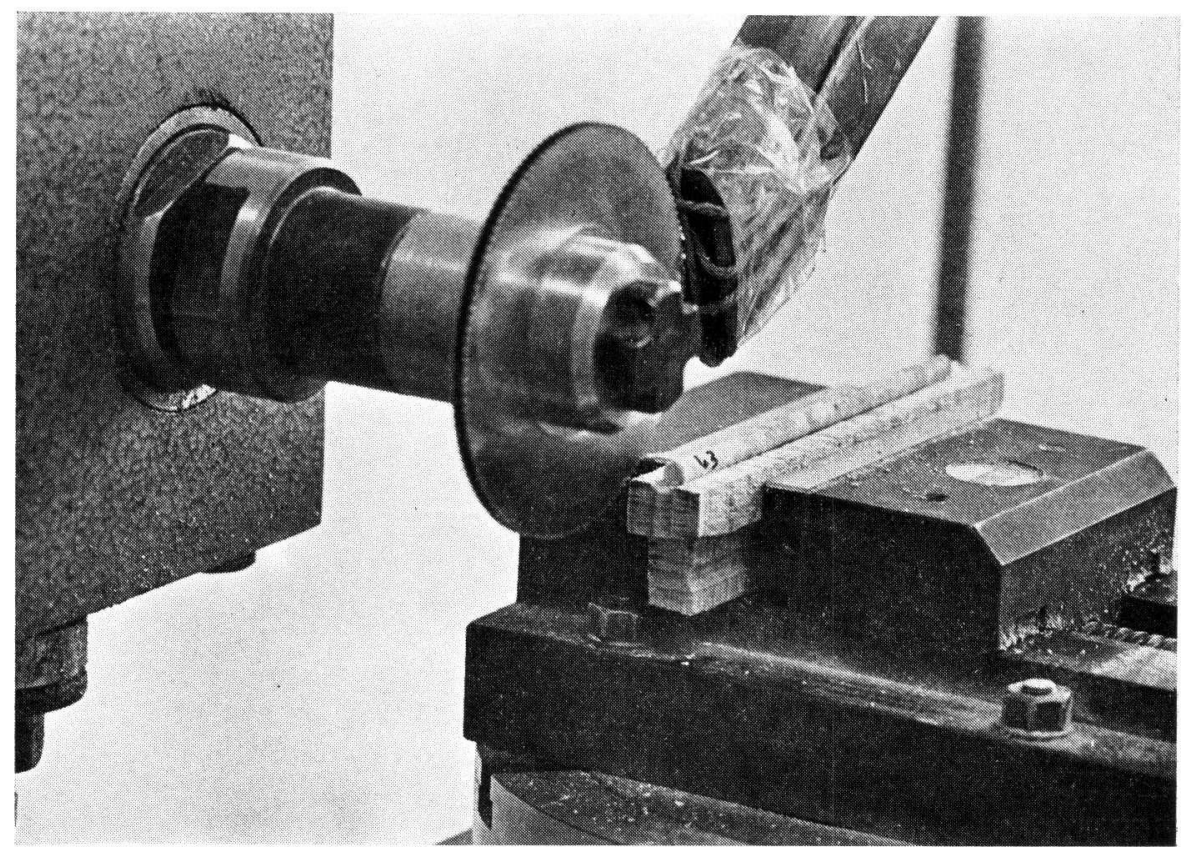

FIG 1. - Débit d'une carotte de sondage collée dans sa gouttière.
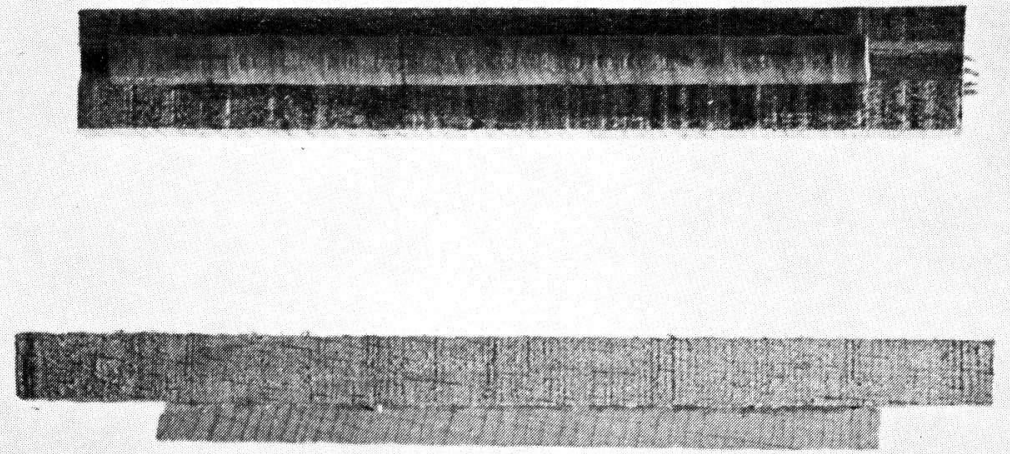

FIG. 2. - Carotte de sondage de hêtre avant et après sciage (grandeur nature). (Noter que la surface de l'échantillon est lissée de façon telle durant le débit que les cernes, très difficiles à distinguer dans cette essence, sont parfaitement visibles). 
- en radiographie axiale, qui est de règle, rappelons-le, pour les espèces à bois homogène, les accroissements annuels sont en principe parallèles les uns aux autres, en sorte que l'on peut en général en obtenir des images nettes sur toute la longueur des échantillons. Il arrive cependant, lorsque l'on a affaire à des carottes de grande longueur, que de légers changements de direction du fil du bois se produisent au cours de la vie de l'arbre (c'est le cas notamment des individus flexueux dans leur jeune âge et qui deviennent de plus en plus rectilignes en vieillissant); il en résulte un risque de flou sur une partie plus ou moins importante de la carotte, flou qui est directement proportionnel à l'épaisseur traversée et qui peut donc être réduit presque à volonté en débitant dans les carottes des échantillons de plus faible épaisseur.

- quant aux radiographies tangentielles (qui s'imposent en particulier pour les feuillus à zone poreuse), elles fournissent également des images qui peuvent être floues pour les accroissements annuels situés près de la moelle, dès lors que les prélèvements ne sont pas parfaitement radiaux; là encore, la radiographie d'échantillons minces débités dans les carottes permet de supprimer presque complètement cet inconvénient.

Qu'il s'agisse de radiographies axiales ou tangentielles, les dimensions des carottes de sondage standards dans ces deux directions sont trop réduites pour qu'il soit possible de les maintenir directement dans l'étau fixé sur le chariot du tour; il convient donc de les assujettir au préalable sur un support intermédiaire constitué par des gouttières en bois dont le rayon correspond à celui des carottes elles-mêmes. Ces gouttières peuvent être fabriquées très rapidement et en grandes séries à l'aide d'un fer de toupie de forme appropriée; les carottes sont enduites de colle à bois sur une de leurs génératrices et serrées dans les gouttières à l'aide de presses en bois qui peuvent recevoir plu-

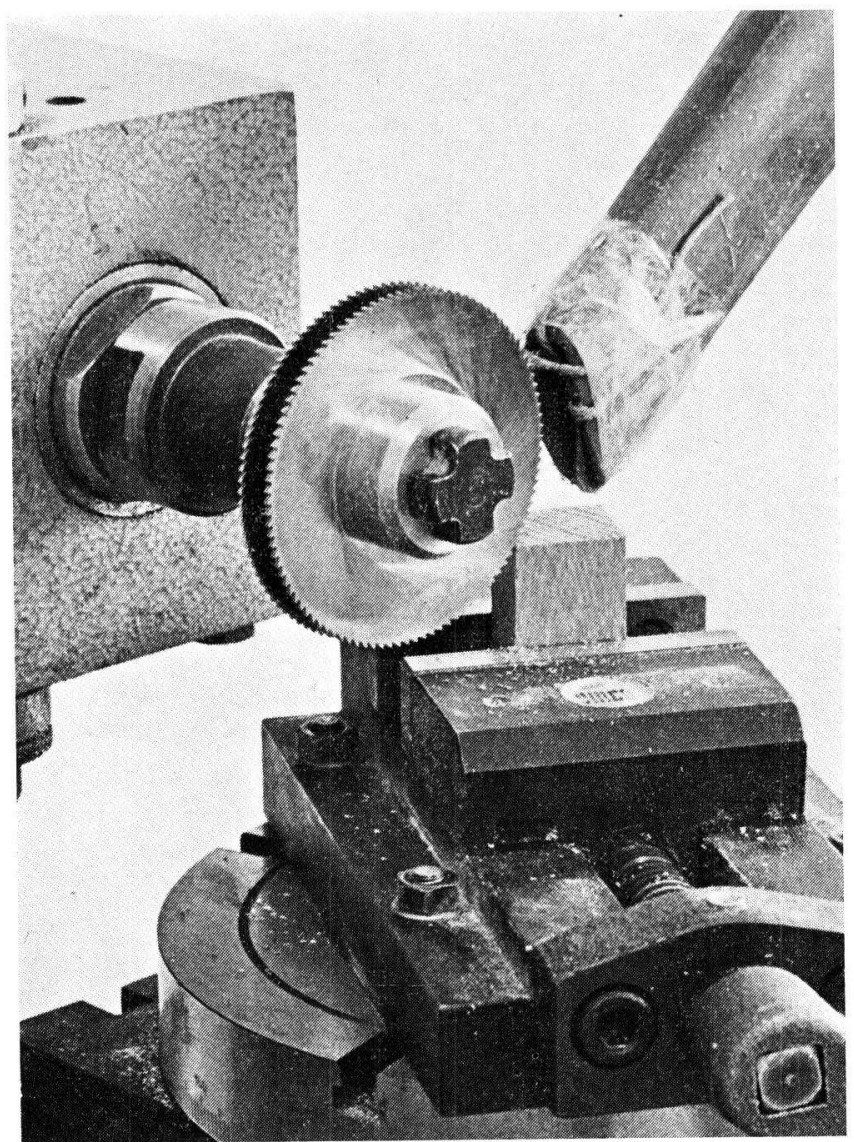

FIG. 3. - Débit d'une barrette $5 \mathrm{~mm} \times 5 \mathrm{~mm}$ dans une éprouvette normalisée. $l^{\text {er }}$ passage : coupe longitudinale radiale. 
sieurs gouttières simultanément. Le positionnement des carottes est fonction tout naturellement du débit, soit tangentiel, soit axial, que l'on souhaite réaliser. Les échantillons sont ensuite sciés comme indiqué ci-dessus, de façon à obtenir des éprouvettes dont l'épaisseur peut être, suivant les cas, de $0,5,1,1,5$ ou $2 \mathrm{~mm}$.

\section{II. - Cas des échantillons de forme parallélépipédique : (figures 3, 4 et 5)}

Le problème se présente de façon systématique lorsque l'on désire étudier les liaisons entre caractéristiques mécaniques et composantes de la densité; on a alors à débiter, dans des éprouvettes normalisées employées pour les essais de résistance mécanique, les barrettes qui ont en principe $5 \mathrm{~mm}$ d'épaisseur, par analogie avec le diamètre des carottes de sondage utilisées pour les analyses de routine. Suivant les cas, cette épaisseur de $5 \mathrm{~mm}$ doit être obtenue dans le sens tangentiel ou dans le sens axial et parfois dans les deux directions simultanément; mais, en toute hypothèse, la grande dimension des barrettes doit correspondre d'une façon aussi rigoureuse que possible à la direction radiale de l'arbre; un morceau d'éprouvette normalisée est à cette fin placé sur la tourelle orientable du chariot qui est positionnée de façon que la direction radiale soit parallèle au sens du déplacement. Un premier passage sous la scie jumelée est alors effectué, puis l'échantillon est réinséré dans l'étau en position perpendiculaire, c'est-à-dire avec le fil du bois normal au plan de sciage; on obtient ainsi des échantillons de section carrée de $5 \mathrm{~mm} \times 5 \mathrm{~mm}$ avec une précision de $\pm 0,01 \mathrm{~mm}$.

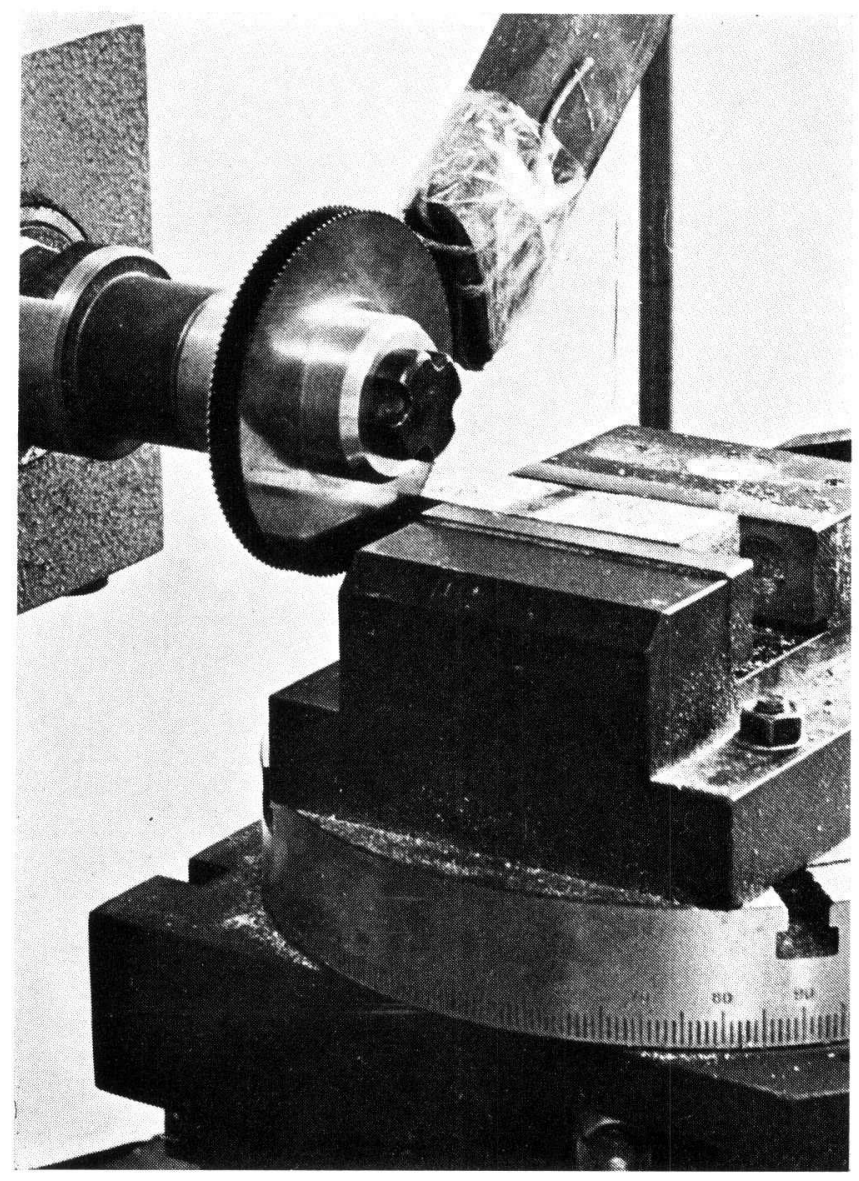

FIG. 4. - 2e passage : coupe transversale. 


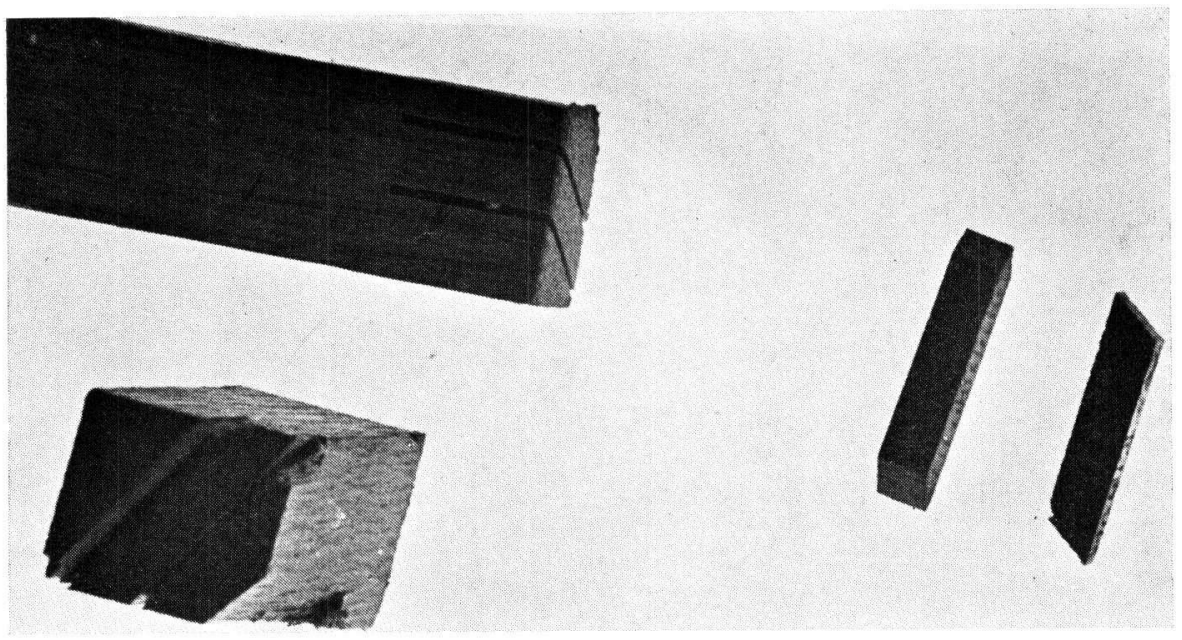

FIG 5. - A gauche en haut : aspect de l'éprouvette normalisée après le ler passage. A gauche en bas: après le $2^{\mathrm{e}}$ passage.

A droite: barrette de $5 \mathrm{~mm} \times 5 \mathrm{~mm}$ obtenue - vue tangentielle et vue axiale, (grandeur nature).

Reç pour publication en mai 1972. 